\title{
ON THE SEMIADDITIVITY \\ OF THE CAPACITIES ASSOCIATED WITH SIGNED VECTOR VALUED RIESZ KERNELS
}

\author{
LAURA PRAT
}

\begin{abstract}
The aim of this paper is to show the semiadditivity of the capacities associated with the signed vector valued Riesz kernels of homogeneity $-\alpha$ in $\mathbb{R}^{n}, 0<\alpha<n$.
\end{abstract}

\section{INTRODUCTION}

In this paper we study the capacity $\gamma_{\alpha}$ related to the signed vector valued Riesz kernels $x /|x|^{1+\alpha}$ in $\mathbb{R}^{n}, 0<\alpha<n$. If $E \subset \mathbb{R}^{n}$ is a compact set and $0<\alpha<n$, one sets

$$
\gamma_{\alpha}(E)=\sup |\langle T, 1\rangle|,
$$

where the supremum is taken over all real distributions $T$ supported on $E$ such that $x_{i} /|x|^{1+\alpha} * T$ is a function in $L^{\infty}\left(\mathbb{R}^{n}\right)$ and $\left\|x_{i} /|x|^{1+\alpha} * T\right\|_{\infty} \leq 1$, for $1 \leq i \leq n$.

When $n=2$ and $\alpha=1$, by the celebrated result of X. Tolsa [T1, $\gamma_{1}$ is basically analytic capacity. Recall that the analytic capacity of a compact set $E \subset \mathbb{C}$ is defined as

$$
\gamma(E)=\sup |\langle T, 1\rangle|,
$$

the supremum taken over all complex distributions $T$ supported on $E$ whose Cauchy potential $1 / z * T$ is a bounded function and $\|1 / z * T\|_{\infty} \leq 1$.

The case $\alpha=n-1, n \geq 2$, is also particularly relevant, because $\gamma_{n-1}$ coincides with the Lipschitz harmonic capacity, introduced in $\left[\mathrm{P}\right.$ to study problems of $\mathcal{C}^{1}$. approximation by harmonic functions in $\mathbb{R}^{n}$ (see also [MaP] and [V]). Notice that the fact that, in the plane, analytic capacity and $\gamma_{1}$ (Lipschitz harmonic capacity) are comparable cannot be deduced just by an inspection of (11) and (2). The reason is that the distributions involved in the supremum in (2) are complex.

In Pr1 one discovered the fact that if $0<\alpha<1$, then a compact set of finite $\alpha$-dimensional Hausdorff measure has zero $\gamma_{\alpha^{-}}$capacity. This is in strong contrast with the situation for integer $\alpha$, in which $\alpha$-dimensional smooth hypersurfaces have positive $\gamma_{\alpha}$ capacity (see $[\mathrm{MaP}]$ ). The case of non-integer $\alpha>1$ is not well understood, although it was shown in Pr1 that for Ahlfors-David regular sets the above-mentioned result (for $0<\alpha<1$ ) still holds in this case (see also [Pr2]).

In MPrV1, the surprising equivalence between $\gamma_{\alpha}, 0<\alpha<1$, and one of the well-known Riesz capacities of non-linear potential theory (see $\mathrm{AH}$, Chapter 1, p. 38]) was established. It was shown that for some positive constant $C$,

$$
C^{-1} C_{\frac{2}{3}(n-\alpha), \frac{3}{2}}(E) \leq \gamma_{\alpha}(E) \leq C C_{\frac{2}{3}(n-\alpha), \frac{3}{2}}(E) .
$$

Received by the editors July 15, 2010.

2010 Mathematics Subject Classification. Primary 42B20. 
Recall that the Riesz capacity $C_{s, p}$ of a compact set $E \subset \mathbb{R}^{n}, 1<p<\infty$, $0<s p \leq n$, is defined by

$$
C_{s, p}(E)=\inf \left\{\|\varphi\|_{p}^{p}: \varphi * \frac{1}{|x|^{n-s}} \geq 1 \text { on } E\right\},
$$

where the infimum is taken over all compactly supported infinitely differentiable functions on $\mathbb{R}^{n}$. The capacity $C_{s, p}$ plays a central role in understanding the nature of Sobolev spaces (see $\mathrm{AH}$ ).

In ENVO it has been shown that the first inequality in (3) holds for all indices $0<\alpha<n$. The opposite inequality is false when $\alpha \in \mathbb{Z}$; for example, if one takes $E$ contained in an $\alpha$-plane with positive $\alpha$-dimensional Hausdorff measure, then $\gamma_{\alpha}(E)>0$ while $C_{\frac{2}{3}(n-\alpha), \frac{3}{2}}(E)=0$. It is an open problem to prove (or disprove) the second inequality in (3) for non-integer $1<\alpha<n$.

Since $C_{s, p}$ is a subadditive set function, as a direct consequence of (3) one gets that $\gamma_{\alpha}$ is semiadditive, that is, given compact sets $E_{1}$ and $E_{2}$,

$$
\gamma_{\alpha}\left(E_{1} \cup E_{2}\right) \leq C\left(\gamma_{\alpha}\left(E_{1}\right)+\gamma_{\alpha}\left(E_{2}\right)\right),
$$

for some constant $C$ depending only on $n$ and $\alpha$. In fact $\gamma_{\alpha}$ is countably semiadditive. In this paper we will show that the semiadditivity of $\gamma_{\alpha}$ holds for all indices $0<\alpha<n$ (see Corollary 2 below).

If we restrict the supremum in (1) to distributions $T$ given by positive Radon measures supported on $E$, we obtain the capacities $\gamma_{\alpha,+}$. Clearly, we have

$$
\gamma_{\alpha,+}(E) \leq \gamma_{\alpha}(E) \text {. }
$$

The arguments of this paper will prove that, in fact, these two quantities are comparable, namely

Theorem 1. There exists an absolute constant $C>0$ such that for any compact set $E \subset \mathbb{R}^{n}$ and any $0<\alpha<n$,

$$
\gamma_{\alpha}(E) \leq C \gamma_{\alpha,+}(E)
$$

This was first shown for $\alpha=1$ and $n=2$ by X. Tolsa [T1, and it was extended to the case $\alpha=n-1$ by Volberg $[\mathrm{Vo}$. For values of $\alpha \in(0,1)$ the result appears in MPrV1]. In [MT, Theorem 1 was proven for a certain class of Cantor sets in $\mathbb{R}^{n}$ (see also T3, where it is proven for a wider class of Cantor sets). Recently, EVo have proven that this comparability result also holds on some examples of random Cantor sets.

As a corollary from Theorem 1, one deduces that $\gamma_{\alpha}$ is countably semiadditive for $0<\alpha<n$.

Corollary 2. Let $E \subset \mathbb{R}^{n}$ be a compact set. Let $E_{i}, i \geq 1$, be Borel sets such that $E=\bigcup_{i=1}^{\infty} E_{i}$. Then,

where $C$ is an absolute constant.

$$
\gamma_{\alpha}\left(\bigcup_{i=1}^{\infty} E_{i}\right) \leq C \sum_{i=1}^{\infty} \gamma_{\alpha}\left(E_{i}\right),
$$

The paper is organized as follows. In Section 2 we prove Corollary 2. In Section 3 we deal with one of the main ingredients for the proof of Theorem 1, a localization $L^{\infty}$-estimate for the scalar kernels $x_{i} /|x|^{1+\alpha}, 0<\alpha<n, 1 \leq i \leq n$. In Section 4 we prove that the capacities $\gamma_{\alpha}$ satisfy an exterior regularity property that will be used for the proof of Theorem 1. Finally, in the last section, we present a sketch of the 
proof of Theorem 1. It becomes clear that the proof depends on the following three facts: a localization $L^{\infty}$ estimate for the $\alpha$-Riesz kernels, the exterior regularity property of $\gamma_{\alpha}, 0<\alpha<n$, and Volberg's extension [Vo] of Tolsa's proof of the semiadditivity of analytic capacity [T1].

Our notation and terminology are standard. For example, $\mathcal{C}_{0}^{\infty}(E)$ denotes the set of all infinitely differentiable functions with compact support contained in the set $E$. Cubes will always be supposed to have sides parallel to the coordinate axis, $l(Q)$ is the side length of the cube $Q$ and $|Q|=l(Q)^{n}$ its volume.

Throughout the paper, the letters $c, C$ will stand for absolute constants depending only on $n$ and $\alpha$ that may change at different occurrences.

\section{Proof of Corollary 2}

In this section we will deduce the semiadditivity of the $\gamma_{\alpha}$ capacity, $0<\alpha<n$, from Theorem 1. For this, we need to introduce the $\alpha$-Riesz transform with respect to an underlying positive Radon measure $\mu$ satisfying the $\alpha$-growth condition

$$
\mu(B(x, r)) \leq C r^{\alpha}, x \in \mathbb{R}^{n}, r \geq 0 .
$$

Given $\varepsilon>0$ we define the truncated $\alpha$-Riesz transform at level $\varepsilon$ as

$$
R_{\varepsilon}(f \mu)(x)=\int_{|y-x|>\varepsilon} \frac{x-y}{|x-y|^{1+\alpha}} f(y) d \mu(y), x \in \mathbb{R}^{n},
$$

for $f \in L^{2}(\mu)$. The growth condition on $\mu$ insures that each $R_{\varepsilon}$ is a bounded operator on $L^{2}(\mu)$ with operator norm $\left\|R_{\varepsilon}\right\|_{L^{2}(\mu)}$ possibly depending on $\varepsilon$. We say that the $\alpha$-Riesz transform is bounded on $L^{2}(\mu)$ when

$$
\|R\|_{L^{2}(\mu)}=\sup _{\varepsilon}\left\|R_{\varepsilon}\right\|_{L^{2}(\mu)}<\infty,
$$

or, in other words, when the truncated $\alpha$-Riesz transforms are uniformly bounded on $L^{2}(\mu)$. Call $L_{\alpha}(E)$ the set of positive Radon measures supported on $E$ which satisfy (5) with $C=1$. One defines $\gamma_{\alpha, o p}(E)$ by

$$
\gamma_{\alpha, o p}(E)=\sup \left\{\mu(E): \mu \in L_{\alpha}(E) \text { and }\|R\|_{L^{2}(\mu)} \leq 1\right\} .
$$

As is well known, the capacities $\gamma_{\alpha,+}(E)$ and $\gamma_{\alpha, o p}(E)$ are comparable; that is, for some positive constant $C$ one has

$$
C^{-1} \gamma_{\alpha, o p}(E) \leq \gamma_{\alpha,+}(E) \leq C \gamma_{\alpha, o p}(E)
$$

for each compact set $E \subset \mathbb{R}^{n}$ (see Lemma 3 in [Pr2]).

Hence, once Theorem 1 is available, namely the fact that $\gamma_{\alpha}(E)$ is comparable to $\gamma_{\alpha,+}(E)$, the semiadditivity of $\gamma_{\alpha}$ holds because $\gamma_{\alpha, o p}$ is obviously semiadditive.

\section{Localization of $\alpha$-Riesz Potentials}

3.1. A growth condition. Let $T$ be a compactly supported distribution in $\mathbb{R}^{n}$ and $0<\alpha<n$. Write $\alpha=[\alpha]+\{\alpha\}$, with $[\alpha] \in \mathbb{Z}$ and $0 \leq\{\alpha\}<1$. We say that the distribution $T$ has growth $\alpha$ provided that

$$
G_{\alpha}(T)=\sup _{\varphi_{Q}} \frac{\left|\left\langle T, \varphi_{Q}\right\rangle\right|}{l(Q)^{\alpha}}<\infty,
$$

where the supremum is taken over all $\varphi_{Q} \in \mathcal{C}_{0}^{\infty}(Q)$ satisfying the normalization inequalities

$$
\left\|\nabla^{n-\alpha} \varphi_{Q}\right\| \leq l(Q)^{\alpha}
$$


where $\left\|\nabla^{n-\alpha} \varphi_{Q}\right\|$ is defined as follows:

(1) For $\alpha=[\alpha] \in \mathbb{Z}$, condition (9) means that

$$
\left\|\nabla^{n-\alpha} \varphi_{Q}\right\|:=\sup _{|s|=n-\alpha}\left\|\partial^{s} \varphi_{Q}\right\|_{L^{1}(Q)} \leq C l(Q)^{\alpha} .
$$

(2) For $\{\alpha\}>0$, condition (9) means that

$$
\left\|\nabla^{n-\alpha} \varphi_{Q}\right\|:=\sup _{|s|=n-[\alpha]}\left\|\partial^{s} \varphi_{Q} * \frac{1}{|x|^{n-\{\alpha\}}}\right\|_{L^{1}} \leq C l(Q)^{\alpha} .
$$

Here we are adopting the standard notation related to multi-indexes, that is, $s=\left(s_{1}, s_{2}, \cdots, s_{n}\right)$, where each coordinate $s_{j}$ is a non-negative integer and $|s|=$ $s_{1}+\cdots+s_{n}$.

For a compact set $E$ in $\mathbb{R}^{n}$ we define $g_{\alpha}(E)$ as the set of all distributions $T$ supported on $E$ having growth $\alpha$ with constant $G_{\alpha}(T)$ at most 1 .

We start by showing that the usual $\alpha$-growth condition for a positive Radon measure (see (5) ) is equivalent to the notion of growth $\alpha$ for distributions, as defined in (8).

Given a positive Radon measure $\mu$ set

$$
L_{\alpha}(\mu)=\sup _{Q} \frac{\mu(Q)}{l(Q)^{\alpha}}
$$

where the supremum is taken over all cubes $Q$ with sides parallel to the coordinate axis.

If $\varphi \in \mathcal{C}_{0}^{\infty}(Q)$, then by an inequality of Maz'ya [MzS, p. 15 and p. 134 ],

$$
|\langle\mu, \varphi\rangle|=\left|\int \varphi d \mu\right| \leq \int|\varphi| d \mu \leq C L_{\alpha}(\mu)\left\|\nabla^{n-\alpha} \varphi\right\| .
$$

Thus, $G_{\alpha}(\mu) \leq C L_{\alpha}(\mu)$.

For the reverse inequality, given a cube $Q$, let $\varphi_{Q}$ be a function in $\mathcal{C}_{0}^{\infty}(2 Q)$ such that $1 \leq \varphi_{Q}$ on $\mathrm{Q}$ and $\left\|\partial^{s} \varphi_{Q}\right\|_{\infty} \leq C_{s} l(Q)^{-|s|},|s| \geq 0$. Then (10) clearly holds when $\alpha \in \mathbb{Z}$. For $\{\alpha\}>0$ and $|s|=n-[\alpha]$, write $|r|=n-[\alpha]-1$. Bringing one derivative from $\partial^{s} \varphi_{Q}$ to the kernel to get integrability in $(4 Q)^{c}$ and using Fubini we obtain

$$
\begin{aligned}
\| \partial^{s} \varphi_{Q} * \frac{1}{|x|^{n-\{\alpha\}} \|_{L^{1}}=} & \int_{(4 Q)^{c}}\left(\partial^{s} \varphi_{Q} * \frac{1}{|x|^{n-\{\alpha\}}}\right)(y) d y \\
& +\int_{4 Q}\left(\partial^{s} \varphi_{Q} * \frac{1}{|x|^{n-\{\alpha\}}}\right)(y) d y \\
\leq & \frac{C}{l(Q)^{|r|}} \int_{(4 Q)^{c}} \int_{Q} \frac{d y d x}{|y-x|^{n+1-\{\alpha\}}} \\
& +\frac{C}{l(Q)^{|s|}} \int_{4 Q} \int_{Q} \frac{d y d x}{|y-x|^{n-\{\alpha\}}} \\
\leq & C l(Q)^{\alpha} .
\end{aligned}
$$

Thus,

Therefore,

$$
\left\|\nabla^{n-\alpha} \varphi_{Q}\right\|:=\sup _{n-[\alpha]}\left\|\partial^{s} \varphi_{Q} * \frac{1}{|x|^{n-\{\alpha\}}}\right\|_{L^{1}} \leq C l(Q)^{\alpha} .
$$

$$
\mu(Q) \leq \int \varphi_{Q} d \mu \leq\left|\left\langle\mu, \varphi_{Q}\right\rangle\right| \leq C G_{\alpha}(\mu) l(Q)^{\alpha}
$$


The next lemma shows that all distributions admissible in the definition of $\gamma_{\alpha}(E)$ have growth $\alpha$.

Lemma 3. Let $T$ be a distribution supported on the compact set $E \subset \mathbb{R}^{n}$. Let $0<\alpha<n$ and suppose that $T$ has bounded $\alpha$-Riesz potential $x /|x|^{1+\alpha} * T$. Then $T \in g_{\alpha}(E)$.

Proof. Our proof uses a reproduction formula for test functions involving the kernel $x_{i} /|x|^{1+\alpha}$, which was first introduced in [Pr1, Lemma 3.1]. There are many variants of this formula depending, for instance, on whether the dimension $n$ and the integer part of $\alpha$ are even or odd. We will consider in full detail only the case of odd dimension of the form $n=2 k+1$. We will also assume that $\alpha$ is non-integer and that its integer part is even, of the form $[\alpha]=2 d$. The argument for the other cases follows the same line of reasoning but using the different variants of the corresponding reproduction formula. In our present case, the reproduction formula we use reads as follows:

$$
\varphi(x)=c \sum_{i=1}^{n} \Delta^{k-d} \partial^{i} \varphi * \frac{x_{i}}{|x|^{1+\alpha}} * \frac{1}{|x|^{n-\{\alpha\}}} .
$$

Let $\varphi_{Q}$ be a $\mathcal{C}_{0}^{\infty}(Q)$ function satisfying the normalization inequalities (9). Then, by (12), the boundedness of the potential $x_{i} /|x|^{1+\alpha} * T, 1 \leq i \leq n$, and Fubini,

$$
\begin{aligned}
\left|\left\langle T, \varphi_{Q}\right\rangle\right| & \leq \sum_{i=1}^{n} \mid\left\langle\frac{x_{i}}{|x|^{1+\alpha}} * T, \Delta^{k-d} \partial_{i} \varphi_{Q} * \frac{1}{\left.|x|^{n-\{\alpha\}}\right\rangle \mid}\right. \\
& \leq C \sum_{i=1}^{n} \int\left|\Delta^{k-d} \partial_{i} \varphi_{Q} * \frac{1}{|x|^{n-\{\alpha\}}}(y)\right| d y \\
& \leq C \sum_{i=1}^{n} \int_{2 Q} \int_{Q} \frac{\left|\Delta^{k-d} \partial_{i} \varphi_{Q}(z)\right|}{|z-y|^{n-\{\alpha\}}} d z d y+C \int_{(2 Q)^{c}} \int_{Q} \frac{\left|\Delta^{k-d} \varphi_{Q}(z)\right|}{|z-y|^{n+1-\{\alpha\}}} d z d y \\
& \leq C l(Q)^{\alpha} .
\end{aligned}
$$

The other cases (namely for odd $n$, even $[\alpha] \ldots$ ) are proven in the same way by using analogous formulae (see [Pr1, Lemma 3.1]).

3.2. Localization of Riesz potentials. When analyzing the argument for the proof of the semiadditivity of analytic capacity (see Theorem 1.1 in [T1]) one realizes that one of the technical tools used is the fact that the Cauchy kernel $1 / z$ localizes in the uniform norm. By this we mean that if $T$ is a compactly supported distribution such that $1 / z * T$ is a bounded measurable function, then $1 / z *(\varphi T)$ is also bounded measurable for each compactly supported $\mathcal{C}^{1}$ function $\varphi$. This is an old result, which is simple to prove because $1 / z$ is related to the differential operator $\bar{\partial}$ (see [G, Chapter V]). The same localization result can be proved easily in any dimension for the kernel $x /|x|^{n}$, which is, modulo a multiplicative constant, the gradient of the fundamental solution of the Laplacian. Again the proof is reasonably straightforward because the kernel is related to a differential operator (see $[\mathrm{P}$. and $[\mathrm{V}]$ ).

In MPrV1, Lemma 3.1] we were concerned with the localization of the vector valued $\alpha$-Riesz kernel $x /|x|^{1+\alpha}, 0<\alpha<n$. For general values of $\alpha$ there is no 
differential operator in the background and consequently the corresponding localization result becomes far from obvious. We now state the general localization lemma proved in MPrV1.

In what follows, given a cube $Q, \varphi_{Q}$ will denote an infinitely differentiable function supported on $Q$ and such that $\left\|\partial^{s} \varphi_{Q}\right\|_{\infty} \leq l(Q)^{-|s|}, 0 \leq|s| \leq n-[\alpha]$.

Lemma 4. Let $T$ be a compactly supported distribution in $\mathbb{R}^{n}$ and let $0<\alpha<n$. Suppose that $x_{i} /|x|^{1+\alpha} * T$ is a bounded measurable function for $1 \leq i \leq n$. Then there exists some constant $C=C(n, \alpha)>0$ such that

$$
\sup _{1 \leq i \leq n}\left\|\frac{x_{i}}{|x|^{1+\alpha}} * \varphi_{Q} T\right\|_{\infty} \leq C \sup _{1 \leq i \leq n}\left\|\frac{x_{i}}{|x|^{1+\alpha}} * T\right\|_{\infty} .
$$

Although Lemma 4 is enough for our purposes, that is to prove Theorem 1, in this paper we will give a proof of a stronger localization result, with a shorter and less technically involved proof. The main difference between the localization lemma in MPrV1 and the one we prove here is that we localize one component of the vector potential $\frac{x}{|x|^{1+\alpha}} * T$, only assuming $L^{\infty}$ estimates on the potential of the same component, instead of assuming $\left\|\frac{x}{|x|^{1+\alpha}} * T\right\|_{\infty} \leq 1$ for the whole vector. Our new localization lemma reads as follows.

Lemma 5. Let $T$ be a compactly supported distribution in $\mathbb{R}^{n}$ with $\alpha$-growth, $0<$ $\alpha<n$, such that $\left(x_{i} /|x|^{1+\alpha}\right) * T$ is in $L^{\infty}\left(\mathbb{R}^{n}\right)$ for some $i, 1 \leq i \leq n$. Then $\left(x_{i} /|x|^{1+\alpha}\right) * \varphi_{Q} T$ is in $L^{\infty}\left(\mathbb{R}^{n}\right)$ and

$$
\left\|\frac{x_{i}}{|x|^{1+\alpha}} * \varphi_{Q} T\right\|_{\infty} \leq C\left(\left\|\frac{x_{i}}{|x|^{2}} * T\right\|_{\infty}+G_{\alpha}(T)\right),
$$

for some positive constant $C=C(n)$ depending only on $n$.

For $\alpha=1$ the proof of the above lemma can be found in [MPrV2]. We remark here that when one deals with indexes $\alpha \in \mathbb{Z}$, the proof of Lemma 5 is less technically involved, since the derivatives $\partial^{s} \varphi_{Q},|s|=n-\alpha$, are ordinary derivatives and therefore supported on the cube $Q$ (compare (11) with (10)).

For the proof of Lemma 5 we need the following result (see Lemma 7 in MPrV2. for the case $\alpha=1$ ), which will be proved after the proof of Lemma 5 .

Lemma 6. Let $T$ be a compactly supported distribution in $\mathbb{R}^{n}$ with $\alpha$-growth, $0<\alpha<n$. Then, for each coordinate $i$, the distribution $\left(x_{i} /|x|^{1+\alpha}\right) * \varphi_{Q} T$ is a locally integrable function and there exists a point $x_{0} \in \frac{1}{4} Q$ such that

$$
\left|\left(\frac{x_{i}}{|x|^{1+\alpha}} * \varphi_{Q} T\right)\left(x_{0}\right)\right| \leq C G_{\alpha}(T),
$$

where $C=C(n)$ is a positive constant depending only on $n$.

Remark. Since the function $f=\left(x_{i} /|x|^{1+\alpha}\right) * \varphi_{Q} T$ is only locally integrable, it may look strange to evaluate $f$ at a point. Indeed, we will show that the mean of $f$ on $\frac{1}{4} Q$ is bounded by $C G_{\alpha}(T)$ and then at many Lebesgue points of $f$ the above stated inequality holds, doubling the constant if necessary.

Proof of Lemma 5. Without loss of generality take $i=1$. We distinguish two cases:

(1) $x \in\left(\frac{3}{2} Q\right)^{c}$. Then $\left|\left(k^{1} * \varphi_{Q} T\right)(x)\right|=\left|\left\langle T, \varphi_{Q}(y) k^{1}(x-y)\right\rangle\right|$. Notice that, for an appropiate dimensional constant $C$, the function

$$
\psi_{Q}(y)=C l(Q)^{\alpha} \varphi_{Q}(y) k^{1}(x-y)
$$


satisfies the normalization inequalities in the definition of $G_{\alpha}(T)$, namely

$$
\left\|\nabla^{n-\alpha} \psi_{Q}\right\| \leq l(Q)^{\alpha} .
$$

Therefore,

$$
\left|\left(k^{1} * \varphi_{Q} T\right)(x)\right|=C l(Q)^{-\alpha}\left|\left\langle T, \psi_{Q}\right\rangle\right| \leq C .
$$

To see (13), observe that if $[\alpha]$ denotes the integer part of $\alpha$ and we write $\alpha=[\alpha]+\{\alpha\}$, then by the Leibniz formula,

$$
\left\|\partial^{s} \psi_{Q}\right\|_{L^{\infty}(Q)} \leq C l(Q)^{\alpha} \sum_{|r|=0}^{|s|} l(Q)^{-|r|} l(Q)^{-|s|-\alpha+|r|} \leq C l(Q)^{-|s|},
$$

for any multi-index $s=\left(s_{1}, \cdots, s_{n}\right)$ with $|s| \geq 0$.

If $\{\alpha\}=0$, (14) immediately implies that condition (13) holds. When $\{\alpha\}>0$, let $s=\left(s_{1}, \cdots, s_{n}\right)$ be any multi-index with $|s|=n-[\alpha]$ and write

$$
\begin{aligned}
\int\left|\left(\partial^{s} \psi_{Q} * \frac{1}{|z|^{n-\{\alpha\}}}\right)(y)\right| d y & =\int_{2 Q}\left|\left(\partial^{s} \psi_{Q} * \frac{1}{|z|^{n-\{\alpha\}}}\right)(y)\right| d y \\
& +\int_{(2 Q)^{c}}\left|\left(\partial^{s} \psi_{Q} * \frac{1}{|z|^{n-\{\alpha\}}}\right)(y)\right| d y=A+B .
\end{aligned}
$$

By (14), we have

$$
A \leq C\left\|\partial^{s} \psi_{Q}\right\|_{\infty} \int_{2 Q} \int_{Q} \frac{d z d y}{|z-y|^{n-\{\alpha\}}} \leq C l(Q)^{\alpha} .
$$

By bringing one derivative from $\partial^{s} \psi_{Q}$ to the kernel $|z|^{-n+\{\alpha\}}$ and using (14) again, we get

$$
\begin{aligned}
& B \leq C \int_{(2 Q)^{c}}\left|\left(\partial^{t} \psi_{Q} * \frac{1}{|z|^{n+1-\{\alpha\}}}\right)(y)\right| d y \\
& \leq C\left\|\partial^{t} \psi_{Q}\right\|_{\infty} \int_{(2 Q)^{c}} \int_{Q} \frac{d z d y}{|z-y|^{n+1-\{\alpha\}}} \leq C l(Q)^{\alpha},
\end{aligned}
$$

for some multi-index $t$ with $|t|=n-[\alpha]-1$.

(2) $x \in \frac{3}{2} Q$. Since $k^{1} * T$ and $\varphi_{Q}$ are bounded functions, we can write

$$
\left|\left(k^{1} * \varphi_{Q} T\right)(x)\right| \leq\left|\left(k^{1} * \varphi_{Q} T\right)(x)-\varphi_{Q}(x)\left(k^{1} * T\right)(x)\right|+\left\|\varphi_{Q}\right\|_{\infty}\left\|k^{1} * T\right\|_{\infty} .
$$

Let $\psi_{Q} \in \mathcal{C}_{0}^{\infty}\left(\mathbb{R}^{n}\right)$ be such that $\psi_{Q} \equiv 1$ in $2 Q, \psi_{Q} \equiv 0$ in $(4 Q)^{c}$ and $\left\|\partial^{s} \psi_{Q}\right\|_{\infty} \leq C_{s} l(Q)^{-|s|}$, for each multi-index $s$. Then one is tempted to write

$$
\begin{aligned}
\left|\left(k^{1} * \varphi_{Q} T\right)(x)-\varphi_{Q}(x)\left(k^{1} * T\right)(x)\right| & \leq\left|\left\langle T, \psi_{Q}(y)\left(\varphi_{Q}(y)-\varphi_{Q}(x)\right) k^{1}(x-y)\right\rangle\right| \\
& +\left\|\varphi_{Q}\right\|_{\infty}\left|\left\langle T,\left(1-\psi_{Q}(y)\right) k^{1}(x-y)\right\rangle\right| .
\end{aligned}
$$

The problem is that the first term on the right-hand side above does not make any sense because $T$ is acting on a function of $y$ which is not necessarily differentiable at the point $x$. To overcome this difficulty one needs to use a standard regularization process. Take $\chi \in \mathcal{C}^{\infty}(B(0,1))$ such that $\int \chi(x) d x=1$ and set $\chi_{\varepsilon}(x)=\varepsilon^{-n} \chi(x / \varepsilon)$. The plan is to estimate, uniformly on $x$ and $\epsilon$,

$$
\left|\left(\chi_{\varepsilon} * k^{1} * \varphi_{Q} T\right)(x)-\varphi_{Q}(x)\left(\chi_{\varepsilon} * k^{1} * T\right)(x)\right| .
$$


Clearly (16) tends, as $\varepsilon$ tends to zero, to

$$
\left|\left(k^{1} * \varphi_{Q} T\right)(x)-\varphi_{Q}(x)\left(k^{1} * T\right)(x)\right|,
$$

for almost all $x \in \mathbb{R}^{n}$, which allows the transfer of uniform estimates. We now have

$$
\begin{aligned}
\mid\left(\chi_{\varepsilon} * k^{1} * \varphi_{Q} T\right)(x)- & \varphi_{Q}(x)\left(\chi_{\varepsilon} * k^{1} * T\right)(x) \mid \\
\leq & \left|\left\langle T, \psi_{Q}(y)\left(\varphi_{Q}(y)-\varphi_{Q}(x)\right)\left(\chi_{\varepsilon} * k^{1}\right)(x-y)\right\rangle\right| \\
& +\left\|\varphi_{Q}\right\|_{\infty}\left|\left\langle T,\left(1-\psi_{Q}(y)\right)\left(\chi_{\varepsilon} * k^{1}\right)(x-y)\right\rangle\right| \\
= & A_{1}+A_{2},
\end{aligned}
$$

where the last identity is the definition of $A_{1}$ and $A_{2}$. To deal with term $A_{1}$ set

$$
k_{\varepsilon}^{1, x}(y)=\left(\chi_{\varepsilon} * k^{1}\right)(x-y) .
$$

We claim that, for an appropriate dimensional constant $C$, the test function

$$
f(y)=C l(Q)^{\alpha} \psi_{Q}(y)\left(\varphi_{Q}(y)-\varphi_{Q}(x)\right) k_{\varepsilon}^{1, x}(y)
$$

satisfies the normalization inequalities (9) in the definition of $G_{\alpha}(T)$, with $\varphi_{Q}$ replaced by $f$. If this is the case, then

$$
A_{1} \leq C l(Q)^{-\alpha}|\langle T, f\rangle| \leq C G_{\alpha}(T) .
$$

To prove the claim we have to show that

$$
\left\|\nabla^{n-\alpha} f\right\| \leq l(Q)^{\alpha}
$$

We first notice that the regularized kernel $\chi_{\varepsilon} * k^{1}$ satisfies the inequalities $\left|\left(\chi_{\varepsilon} * \partial^{s} k^{1}\right)(x)\right| \leq \frac{C}{|x|^{\alpha+|s|}}, \quad x \in \mathbb{R}^{n} \backslash\{0\} \quad$ and $\quad 0 \leq|s| \leq n-[\alpha]-1$,

where $C$ is a dimensional constant, which, in particular, is independent of $\epsilon$.

The estimate of the $L^{1}$-norm in (17) requires the use of the Leibniz formula

$$
\partial^{s}\left(\psi_{Q}\left(\varphi_{Q}-\varphi_{Q}(x)\right) k_{\varepsilon}^{1, x}\right)=\sum_{|r|=0}^{|s|} c_{r, s} \partial^{r}\left(\psi_{Q}\left(\varphi_{Q}-\varphi_{Q}(x)\right)\right) \partial^{s-r} k_{\varepsilon}^{1, x}
$$

and of (18) . Notice that for any multi-index $t=\left(t_{1}, \cdots, t_{n}\right)$ with $|t|=$ $n-[\alpha]-1$,

$$
\begin{aligned}
\left\|\partial^{t} f\right\|_{L^{1}(4 Q)} & \leq C l(Q)^{\alpha} \sum_{|r|=0}^{|t|} \frac{1}{l(Q)^{|r|}} \int_{4 Q}\left|\partial^{t-r}\left(k_{\varepsilon}^{1, x}\right)(y)\right| d y \\
& \leq C l(Q)^{n-|t|}=C l(Q)^{[\alpha]+1} .
\end{aligned}
$$


If $s=\left(s_{1}, \cdots, s_{n}\right)$ is such that $|s|=n-[\alpha]$, using the mean value theorem to gain integrability when $|r|=0$ and (18),

$$
\begin{aligned}
\left\|\partial^{s} f\right\|_{L^{1}(4 Q)} \leq & C l(Q)^{\alpha}\left\|\nabla \varphi_{Q}\right\|_{\infty} \int_{4 Q} \frac{d y}{|y-x|^{\alpha+|s|-1}} \\
& +C l(Q)^{\alpha} \sum_{|r|=1}^{|s|} \frac{1}{l(Q)^{|r|}} \int_{4 Q}\left|\partial^{s-r}\left(k_{\varepsilon}^{1, x}\right)(y)\right| d y \\
\leq & C l(Q)^{[\alpha]} .
\end{aligned}
$$

Estimate (21) immediately yields (17) for $\{\alpha\}=0$,

$$
\left\|\nabla^{n-\alpha} f\right\|:=\sup _{|s|=n-\alpha} \int_{4 Q}\left|\partial^{s} f(y)\right| d y \leq C l(Q)^{\alpha} .
$$

If $\{\alpha\}>0$, then for any multi-index $s=\left(s_{1}, \cdots, s_{n}\right)$ with $|s|=n-[\alpha]$,

$$
\begin{aligned}
\int\left|\left(\partial^{s} f * \frac{1}{|z|^{n-\{\alpha\}}}\right)(y)\right| d y \leq & \int_{5 Q} \mid\left(\partial^{s} f * \frac{1}{\left.|z|^{n-\{\alpha\}}\right)(y) \mid d y}\right. \\
& +C \int_{(5 Q)^{c}}\left|\left(\partial^{t} f * \frac{1}{|z|^{n+1-\{\alpha\}}}\right)(y)\right| d y \\
= & B_{1}+B_{2},
\end{aligned}
$$

where $t$ is some multi-index with $|t|=n-[\alpha]-1$.

To estimate $B_{2}$, we use Fubini and (20). Then,

$$
B_{2} \leq \int_{4 Q}\left|\partial^{t} f(z)\right| \int_{(5 Q)^{c}} \frac{d y d z}{|z-y|^{n+1-\{\alpha\}}} \leq C l(Q)^{\alpha} .
$$

We turn now to term $B_{1}$. By Fubini and (21), we get

$$
B_{1} \leq C \int_{5 Q}\left|\partial^{s} f(z)\right| \int_{4 Q} \frac{d y d z}{|y-z|^{n-\{\alpha\}}} \leq C l(Q)^{\alpha} .
$$

This finishes the proof of (17). We now turn to $A_{2}$. By Lemma 6 , there exists a point $x_{0} \in Q$ such that $\left|\left(k^{1} * \psi_{Q} T\right)\left(x_{0}\right)\right| \leq C G_{\alpha}(T)$. Then

$$
\left|\left(k^{1} *\left(1-\psi_{Q}\right) T\right)\left(x_{0}\right)\right| \leq C\left(\left\|k^{1} * T\right\|_{\infty}+G_{\alpha}(T)\right) .
$$

Arguing as in the proof of Lemma [6, one can see that the analogous inequality holds as well for the regularized potentials appearing in $A_{2}$, uniformly in $\epsilon$, and therefore

$$
A_{2} \leq C\left|\left\langle T,\left(1-\psi_{Q}\right)\left(k_{\varepsilon}^{1, x}-k_{\varepsilon}^{1, x_{0}}\right)\right\rangle\right|+C\left(\left\|k^{1} * T\right\|_{\infty}+G_{\alpha}(T)\right) .
$$

To estimate $\left|\left\langle T,\left(1-\psi_{Q}\right)\left(k_{\varepsilon}^{1, x}-k_{\varepsilon}^{1, x_{0}}\right)\right\rangle\right|$, we decompose $\mathbb{R}^{n} \backslash\{x\}$ into a union of rings

$$
R_{j}=\left\{z \in \mathbb{R}^{n}: 2^{j} l(Q) \leq|z-x| \leq 2^{j+1} l(Q)\right\}, \quad j \in \mathbb{Z},
$$

and consider functions $\varphi_{j}$ in $\mathcal{C}_{0}^{\infty}\left(\mathbb{R}^{n}\right)$, with support contained in $\frac{3}{2} R_{j}$, such that $\left\|\partial^{s} \varphi_{j}\right\|_{\infty} \leq C\left(2^{j} l(Q)\right)^{-|s|},|s| \geq 0$, and $\sum_{j} \varphi_{j}=1$ on $\mathbb{R}^{n} \backslash\{x\}$. Then, 
since $x \in \frac{3}{2} Q$ and $1-\psi_{Q} \equiv 0$ in $2 Q$, the smallest ring $R_{j}$ that may intersect $(2 Q)^{c}$ is $R_{-2}$. Therefore we have

$$
\begin{aligned}
\left|\left\langle T,\left(1-\psi_{Q}\right)\left(k_{\varepsilon}^{1, x}-k_{\varepsilon}^{1, x_{0}}\right)\right\rangle\right|= & \left|\left\langle T, \sum_{j \geq-2} \varphi_{j}\left(1-\psi_{Q}\right)\left(k_{\varepsilon}^{1, x}-k_{\varepsilon}^{1, x_{0}}\right)\right\rangle\right| \\
\leq & \left|\left\langle T, \sum_{j \in I} \varphi_{j}\left(1-\psi_{Q}\right)\left(k_{\varepsilon}^{1, x}-k_{\varepsilon}^{1, x_{0}}\right)\right\rangle\right| \\
& +\sum_{j \in J}\left|\left\langle T, \varphi_{j}\left(k_{\varepsilon}^{1, x}-k_{\varepsilon}^{1, x_{0}}\right)\right\rangle\right|
\end{aligned}
$$

where $I$ denotes the set of indices $j \geq-2$ such that the support of $\varphi_{j}$ intersects $4 Q$ and $J$ the remaining indices, namely those $j \geq-2$ such that the support of $\varphi_{j}$ is contained in the complement of $4 Q$. Notice that the cardinality of $I$ is bounded by a dimensional constant.

Set

$$
g=C l(Q)^{\alpha} \sum_{j \in I} \varphi_{j}\left(1-\psi_{Q}\right)\left(k_{\varepsilon}^{1, x}-k_{\varepsilon}^{1, x_{0}}\right),
$$

and for $j \in J$,

$$
g_{j}=C 2^{j}\left(2^{j} l(Q)\right)^{\alpha} \varphi_{j}\left(k_{\varepsilon}^{1, x}-k_{\varepsilon}^{1, x_{0}}\right) .
$$

We now show that the test functions $g$ and $g_{j}, j \in J$, satisfy the normalization inequalities (9) in the definition of $G_{\alpha}(T)$. Once this is available, using the $\alpha$-growth condition of $T$ we obtain

$$
\begin{aligned}
\left|\left\langle T,\left(1-\psi_{Q}\right)\left(k_{\varepsilon}^{1, x}-k_{\varepsilon}^{1, x_{0}}\right)\right\rangle\right| \leq & C l(Q)^{-\alpha}|\langle T, g\rangle| \\
& +C \sum_{j \in J} 2^{-j}\left(2^{j} l(Q)\right)^{-\alpha}\left|\left\langle T, g_{j}\right\rangle\right| \\
\leq & C G_{\alpha}(T)+C \sum_{j \geq 2} 2^{-j} G_{\alpha}(T) \leq C G_{\alpha}(T),
\end{aligned}
$$

which completes the proof of Lemma 7.

We now check the normalization inequalities for $g$ and $g_{j}$. For $g$ one argues as in the proof of (17), using that $\left\|\partial^{s}\left(1-\psi_{Q}\right)\right\|_{\infty} \leq C l(Q)^{-|s|}$, $\left\|\partial^{s} \varphi_{j}\right\|_{\infty} \leq C l(Q)^{-|s|}, j \in I$, (18), the fact that $x, x_{0} \in \frac{3}{2} Q, y \in(2 Q)^{c}$, and a gradient estimate.

For $g_{j}$ we use in addition the Leibniz formula and a gradient estimate to show that, for $j \in J$, and $n-[\alpha]-1 \leq|s| \leq n-[\alpha]$,

$$
\begin{aligned}
\left\|\partial^{s} g_{j}\right\|_{\infty} & \leq C 2^{j}\left(2^{j} l(Q)\right)^{\alpha} \sum_{|r|=0}^{|s|} \frac{1}{\left(2^{j} l(Q)\right)^{|r|}} \frac{l(Q)}{\left(2^{j} l(Q)\right)^{1+\alpha+|s|-|r|}} \\
& \leq C\left(2^{j} l(Q)\right)^{-|s|} .
\end{aligned}
$$

If $\{\alpha\}=0$, we use (22) to obtain

$$
\left\|\nabla^{n-\alpha} g_{j}\right\|:=\sup _{|s|=n-\alpha} \int\left|\partial^{s} g_{j}(y)\right| d y \leq C\left(2^{j} l(Q)\right)^{\alpha} .
$$

If $\{\alpha\}>0$, then for any multi-index $\left.s=\left(s_{1}, \cdots, s_{n}\right)\right)$ with $|s|=n-$ $[\alpha]-1$, using Fubini, (22) and arguing similar to the proof of (17) we get, 
for some multi-index $t=\left(t_{1}, \cdots, t_{n}\right)$ with $|t|=n-[\alpha]-1$,

$$
\begin{aligned}
\int\left|\left(\partial^{s} g_{j} * \frac{1}{|z|^{n-\{\alpha\}}}\right)(y)\right| d y \leq & \int_{2 R_{j}}\left|\left(\partial^{s} g_{j} * \frac{1}{|z|^{n-\{\alpha\}}}\right)(y)\right| d y \\
& +C \int_{\left(2 R_{j}\right)^{c}}\left|\left(\partial^{t} g_{j} * \frac{1}{|z|^{n+1-\{\alpha\}}}\right)(y)\right| d y \\
\leq & C\left(2^{j} l(Q)\right)^{\alpha} .
\end{aligned}
$$

Therefore, we can conclude that

$$
\left\|\nabla^{n-\alpha} g_{j}\right\| \leq C\left(2^{j} l(Q)\right)^{\alpha} .
$$

Proof of Lemma 6. Without loss of generality, set $i=1$ and write $k^{1}(x)=$ $x_{1} /|x|^{1+\alpha}$. Since $k^{1} * \varphi_{Q} T$ is infinitely differentiable off the closure of $Q$, we only need to show that $k^{1} * \varphi_{Q} T$ is integrable on $2 Q$. We will actually prove a stronger statement, namely, that $k^{1} * \varphi_{Q} T$ is in $L^{p}(2 Q)$ for each $p$ in the interval $1 \leq p<n$. Indeed, fix any $q$ satisfying $n /(n-1)<q<\infty$ and call $p$ the dual exponent, so that $1<p<n$. We need to estimate the action of $k^{1} * \varphi_{Q} T$ on functions $\psi \in \mathcal{C}_{0}^{\infty}(2 Q)$ in terms of $\|\psi\|_{q}$. We clearly have

$$
\left\langle k^{1} * \varphi_{Q} T, \psi\right\rangle=\left\langle T, \varphi_{Q}\left(k^{1} * \psi\right)\right\rangle .
$$

We claim that, for an appropriate dimensional constant $C$, the test function

$$
\frac{\varphi_{Q}\left(k^{1} * \psi\right)}{C l(Q)^{\frac{n}{p}-\alpha}\|\psi\|_{q}}
$$

satisfies the normalization inequalities (9) in the definition of $G_{\alpha}(T)$. Once this is proved, by the definition of $G_{\alpha}(T)$ we get

$$
\left|\left\langle k^{1} * \varphi_{Q} T, \psi\right\rangle\right| \leq C l(Q)^{\frac{n}{p}}\|\psi\|_{q} G_{\alpha}(T),
$$

and so

$$
\left\|k^{1} * \varphi_{Q} T\right\|_{L^{p}(2 Q)} \leq C l(Q)^{\frac{n}{p}} G_{\alpha}(T)
$$

Hence

$$
\begin{aligned}
\frac{1}{\left|\frac{1}{4} Q\right|} \int_{\frac{1}{4} Q}\left|\left(k^{1} * \varphi_{Q} T\right)(x)\right| d x & \leq 4^{n}\left(\frac{1}{|Q|} \int_{Q}\left|\left(k^{1} * \varphi_{Q} T\right)(x)\right|^{p} d x\right)^{\frac{1}{p}} \\
& \leq C G_{\alpha}(T)
\end{aligned}
$$

which completes the proof of Lemma 8.

To prove the claim we have to show that

$$
\left\|\nabla^{n-\alpha}\left(\varphi_{Q}\left(k^{1} * \psi\right)\right)\right\| \leq C l(Q)^{\frac{n}{p}}\|\psi\|_{q} .
$$

Write $\alpha=[\alpha]+\{\alpha\}$, with $\{\alpha\} \in[0,1)$ and $[\alpha] \in \mathbb{Z}$. We now distinguish two cases, $\{\alpha\}=0$ and $\{\alpha\}>0$.

(1) Case $\{\alpha\}=0$, i.e. $\alpha=[\alpha] \in \mathbb{Z}$. This is the easiest case, because the derivatives appearing in (23) are ordinary derivatives (see also lemma 7 in MPrV2]). 
Let $s=\left(s_{1}, s_{2}, \cdots, s_{n}\right)$ be any multi-index with $|s|=n-\alpha$. Using the Leibniz formula,

$$
\partial^{s}\left(\varphi_{Q}\left(k^{1} * \psi\right)\right)=\sum_{|r|=0}^{|s|} c_{s, r} \partial^{r} \varphi_{Q} \partial^{s-r}\left(k^{1} * \psi\right),
$$

we obtain

$$
\begin{aligned}
\int_{Q}\left|\partial^{s}\left(\varphi_{Q}\left(k^{1} * \psi\right)\right)(y)\right| d y \leq & C \int_{2 Q}\left|\left(\partial^{s} k^{1} * \psi\right)(y)\right| d y \\
& +C \sum_{|r|=1}^{|s|} \int_{Q}\left|\partial^{r} \varphi_{Q}(y)\right|\left|\partial^{s-r}\left(k^{1} * \psi\right)(y)\right| d y=A+B .
\end{aligned}
$$

To estimate term $A$, we remark that for $|s|=n-\alpha$,

$$
\partial^{s} k^{1} * \psi=c \psi+S(\psi)
$$

where $S$ is a smooth homogeneous convolution Calderón-Zygmund operator and $c$ a constant depending on $s$. This can be seen by computing the Fourier transform of $\partial^{s} k^{1}$ and then using that each homogeneous polynomial can be decomposed in terms of homogeneous harmonic polynomials of lower degrees (see [St, p. 69]). Since Calderón-Zygmund operators preserve $L^{q}\left(\mathbb{R}^{n}\right), 1<q<\infty$, we get, using Hölder,

$$
A \leq C l(Q)^{\frac{n}{p}}\|\psi\|_{q} .
$$

To estimate term $B$, we use $\left|\partial^{s-r} k^{1}(x)\right| \leq C|x|^{-(\alpha+|s|-|r|)}$, Fubini, the fact that $\left\|\partial^{r} \varphi_{Q}\right\|_{\infty} \leq C l(Q)^{-|r|}$ and Hölder to obtain $B \leq C l(Q)^{\frac{n}{p}}\|\psi\|_{q}$. Therefore we get

$$
\left\|\nabla^{n-\alpha}\left(\varphi_{Q}\left(k^{1} * \psi\right)\right)\right\|=\sup _{|s|=n-\alpha} \int_{Q}\left|\partial^{s}\left(\varphi_{Q}\left(k^{1} * \psi\right)\right)(y)\right| d y \leq C l(Q)^{\frac{n}{p}}\|\psi\|_{q} .
$$

(2) Case $\{\alpha\}>0$. Let $s=\left(s_{1}, s_{2}, \cdots, s_{n}\right)$ be any multi-index with $|s|=n-\alpha$ and write

$$
\begin{aligned}
\int\left|\partial^{s}\left(\varphi_{Q}\left(k^{1} * \psi\right) * \frac{1}{|x|^{n-\{\alpha\}}}\right)(x)\right| d x= & \int_{(2 Q)^{c}}\left|\left(\partial^{s} \varphi_{Q}\left(k^{1} * \psi\right) * \frac{1}{|x|^{n-\{\alpha\}}}\right)(x)\right| d x \\
& +\int_{2 Q}\left|\partial^{s}\left(\varphi_{Q}\left(k^{1} * \psi\right) * \frac{1}{|x|^{n-\{\alpha\}}}\right)(x)\right| d x \\
= & A+B .
\end{aligned}
$$

We deal first with term $A$. Bringing one derivative from $\partial^{s}\left(\varphi_{Q}\left(k^{1} * \psi\right)\right)$ to the kernel $|x|^{-n+\{\alpha\}}$ and using Fubini, we obtain

$$
\left.A \leq C \int_{(2 Q)^{c}} \mid \partial^{t}\left(\varphi_{Q}\left(k^{1} * \psi\right)\right) * \frac{1}{|x|^{n+1-\{\alpha\}}}\right)(x) \mid d x,
$$

for some multi-index $t=\left(t_{1}, \cdots, t_{n}\right)$ with $|t|=n-[\alpha]-1$.

We will now use the Leibniz formula (24) (with $s$ replaced by $t$ ) and the fact that

$$
\left|\partial^{t-r} k^{1}(x)\right| \leq C|x|^{-(\alpha+|t|-|r|)} .
$$


Therefore, since $\alpha+|t|-|r|<n$, by Fubini and Hölder we obtain

$$
\begin{aligned}
\int_{Q}\left|\partial^{t}\left(\varphi_{Q}\left(k^{1} * \psi\right)\right)(y)\right| d y & \leq C \sum_{|r|=0}^{|t|} \int_{Q}\left|\partial^{r} \varphi_{Q}(y)\right|\left|\partial^{t-r}\left(k^{1} * \psi\right)(y)\right| d y \\
& \leq C \sum_{|r|=0}^{|t|} \int_{2 Q}|\psi(x)| \int_{Q} \frac{\left|\partial^{r} \varphi_{Q}(y)\right|}{|y-x|^{\alpha+|t|-|r|}} d y d x \\
& \leq C\|\psi\|_{q} l(Q)^{\frac{n}{p}+1-\{\alpha\}} .
\end{aligned}
$$

Hence, if we apply Fubini again, we get

$A \leq C \int_{Q}\left|\partial^{t}\left(\varphi_{Q}\left(k^{1} * \psi\right)\right)(y)\right| \int_{(2 Q)^{c}} \frac{d x d y}{|y-x|^{n+1-\{\alpha\}}} \leq C l(Q)^{\frac{n}{p}}\|\psi\|_{q}$.

To estimate term $B$, we will use (24) and for each $0 \leq|r| \leq|t|$ we will add and subtract $\partial^{r} \varphi_{Q}(x) \partial^{t-r}\left(k^{1} * \psi\right)(y)$ in the integral to gain integrability, namely

$$
\begin{aligned}
B \leq & C \sum_{|r|=0}^{|t|} \int_{2 Q}\left|\int_{Q} \frac{\left(\partial^{r} \varphi_{Q}(y)-\partial^{r} \varphi_{Q}(x)\right) \partial^{t-r}\left(k^{1} * \psi\right)(y)}{|y-x|^{n+1-\{\alpha\}}} d y\right| d x \\
& +C \sum_{|r|=0}^{|t|} \int_{Q}\left|\partial^{r} \varphi_{Q}(x) \|\left(\Delta \partial^{t-r} k^{1} * \psi * \frac{1}{|y|^{n-1-\{\alpha\}}}\right)(x)\right| d x \\
& +C \sum_{|r|=0}^{|t|} \int_{Q}\left|\partial^{r} \varphi_{Q}(x) \| \int_{Q^{c}} \frac{\partial^{t-r}\left(k^{1} * \psi\right)(y)}{|y-x|^{n+1-\{\alpha\}}} d y\right| d x \\
= & B_{1}+B_{2}+B_{3},
\end{aligned}
$$

the last identity being a definition for $B_{1}, B_{2}$ and $B_{3}$.

Since arguing as in (26) (recall that $|t|=n-[\alpha]-1$ ), we obtain

$$
\int_{Q}\left|\partial^{t-r}\left(k^{1} * \psi\right)(y)\right| d y \leq\|\psi\|_{q} l(Q)^{\frac{n}{p}+1-\{\alpha\}+|r|},
$$

by the mean value theorem and Fubini, we get that

$$
\begin{aligned}
B_{1} & \leq \sum_{|r|=0}^{|t|} \frac{C}{l(Q)^{|r|+1}} \int_{Q}\left|\partial^{t-r}\left(k^{1} * \psi\right)(y)\right| \int_{2 Q} \frac{d x d y}{|y-x|^{n-\{\alpha\}}} \\
& \leq C \sum_{|r|=0}^{|t|} l(Q)^{-|r|-1}\|\psi\|_{q} l(Q)^{\frac{n}{p}+1-\{\alpha\}+|r|} l(Q)^{\{\alpha\}} \\
& \leq C l(Q)^{\frac{n}{p}}\|\psi\|_{q} .
\end{aligned}
$$

We now deal with term $B_{2}$. By computing the Fourier transform of the convolution $\Delta \partial^{t-r} k^{1} * \psi * \frac{1}{|y|^{n-1-\{\alpha\}}}$, one can see that for $|r|=0$,

$$
\left(\Delta \partial^{t} k^{1} * \psi * \frac{1}{|y|^{n-1-\{\alpha\}}}\right)(x)=c \psi+c S_{0}(\psi)(x),
$$


where $c$ is a constant and $S_{0}$ is a smooth homogeneous convolution CalderónZygmund operator. For $|r| \geq 1$, we obtain

$$
\left(\Delta \partial^{t-r} k^{1} * \psi * \frac{1}{|y|^{n-1-\{\alpha\}}}\right)(x)=c S_{r}(\psi)(x),
$$

with $S_{r}$ a convolution Calderón-Zygmund operator with kernel of homogeneity $-(n-|r|)$. Since Calderón-Zygmund operators preserve $L^{q}\left(\mathbb{R}^{n}\right)$, $1<q<\infty$, using Young's inequality to estimate the $L^{q}(Q)$-norm of the convolution $S_{r}(\psi)$ and Hölder, we obtain

$$
\begin{aligned}
B_{2} & =\sum_{|r|=0}^{|t|} \int_{Q}\left|\partial^{r} \varphi_{Q}(x) \| S_{r}(\psi)(x)\right| d x \\
& \leq C\left\|\varphi_{Q}\right\|_{p}\|\psi\|_{q}+C \sum_{|r|=1}^{|t|}\left\|\partial^{r} \varphi_{Q}\right\|_{p}\left\|S_{r}(\psi)\right\|_{L^{q}(Q)} \\
& \leq C l(Q)^{\frac{n}{p}}\|\psi\|_{q}+\|\psi\|_{q} \sum_{|r|=1}^{|t|} l(Q)^{-|r|} l(Q)^{\frac{n}{p}} l(Q)^{|r|} \leq C l(Q)^{\frac{n}{p}}\|\psi\|_{q} .
\end{aligned}
$$

Now we are only left with term $B_{3}$. Since $\partial^{r} \varphi_{Q}$ is supported on $Q$, we can subtract $\partial^{r} \varphi_{Q}(y)=0$ for $y \in 3 Q \backslash Q,|r| \geq 0$. Then,

$$
\begin{aligned}
B_{3}= & \int_{Q}\left|\int_{Q^{c}} \partial^{r} \varphi_{Q}(x) \frac{\partial^{t-r}\left(k^{1} * \psi\right)(y)}{|y-x|^{n+1-\{\alpha\}}} d y\right| d x \\
\leq & \sum_{|r|=0}^{|t|} \int_{Q}\left|\int_{3 Q \backslash Q} \partial^{t-r}\left(k^{1} * \psi\right)(y) \frac{\partial^{r} \varphi_{Q}(x)-\partial \varphi_{Q}(y)}{|y-x|^{n+1-\{\alpha\}}} d y\right| d x \\
& +\sum_{|r|=0}^{|t|} \int_{Q}\left|\int_{(3 Q)^{c}} \partial^{r} \varphi_{Q}(x) \frac{\partial^{t-r}\left(k^{1} * \psi\right)(y)}{|y-x|^{n+1-\{\alpha\}}} d y\right| d x=B_{31}+B_{32} .
\end{aligned}
$$

Using the mean value theorem and proceeding as in (27),

$$
B_{31} \leq \sum_{|r|=0}^{|t|} \frac{C}{l(Q)^{|r|+1}} \int_{Q} \int_{3 Q} \frac{\left|\partial^{t-r}\left(k^{1} * \psi\right)(y)\right|}{|y-x|^{n-\{\alpha\}}} d y d x \leq C l(Q)^{\frac{n}{p}}\|\psi\|_{q} .
$$

Notice that by (25), for $y \in(3 Q)^{c}$,

$$
\left|\partial^{t-r}\left(k^{1} * \psi\right)(y)\right| \leq \int_{2 Q} \frac{\psi(z)}{|z-y|^{\alpha+|t|-|r|}} d z \leq C\|\psi\|_{q} l(Q)^{\frac{n}{p}} l(Q)^{-\alpha-|t|+|r|} .
$$

Therefore,

$$
\begin{aligned}
B_{32} & \leq \sum_{|r|=0}^{|t|} \frac{C}{l(Q)^{|r|}} \int_{Q} \int_{(3 Q)^{c}} \frac{\left|\partial^{t-r}\left(k^{1} * \psi\right)(y)\right|}{|y-x|^{n+1-\{\alpha\}}} d y d x \\
& \leq c\|\psi\|_{q} l(Q)^{\frac{n}{p}} \sum_{|r|=0}^{|t|} \frac{l(Q)^{-\alpha-|t|+|r|}}{l(Q)^{|r|}} \int_{Q} \int_{(3 Q)^{c}} \frac{1}{|y-x|^{n+1-\{\alpha\}}} d y d x \\
& \leq C l(Q)^{\frac{n}{p}}\|\psi\|_{q},
\end{aligned}
$$

which finishes the proof of (23) for the case $\{\alpha\}>0$. 


\section{A Continuity PRoperty For the CApacity $\gamma$}

In this section we prove a continuity property for the capacity $\gamma_{\alpha}, 0<\alpha<n$, which will be used in the proof of Theorem 1.

Lemma 7. Let $\left\{E_{j}\right\}_{j}$ be a decreasing sequence of compact sets, with intersection the compact set $E \subset \mathbb{R}^{n}$ and let $0<\alpha<n$. Then

$$
\gamma_{\alpha}(E)=\lim _{j \rightarrow \infty} \gamma_{\alpha}\left(E_{j}\right)
$$

Proof. Since, by definition, the set function $\gamma_{\alpha}$ is non-decreasing,

$$
\lim _{j \rightarrow \infty} \gamma_{\alpha}\left(E_{j}\right) \geq \gamma_{\alpha}(E),
$$

and the limit clearly exists. For each $j \geq 1$, let $T_{j}$ be a distribution such that the potential $x /|x|^{1+\alpha} * T_{j}$ is in the unit ball of $L^{\infty}\left(\mathbb{R}^{n}\right)$, and

$$
\gamma_{\alpha}\left(E_{j}\right)-\frac{1}{j}<\left|\left\langle T_{j}, 1\right\rangle\right| \leq \gamma_{\alpha}\left(E_{j}\right)
$$

We want to show that for each test function $\varphi$,

$$
\left\langle T_{j}, \varphi\right\rangle \underset{j \rightarrow \infty}{\longrightarrow}\langle T, \varphi\rangle,
$$

for some distribution $T$ whose potential $x /|x|^{1+\alpha} * T$ is in the unit ball of $L^{\infty}\left(\mathbb{R}^{n}\right)$. If (28) holds and $\varphi$ is a test function satisfying $\varphi \equiv 1$ in a neighbourhood of $E$, then

$$
\lim _{j \rightarrow \infty} \gamma_{\alpha}\left(E_{j}\right)=\lim _{j \rightarrow \infty}\left|\left\langle T_{j}, 1\right\rangle\right|=\lim _{j \rightarrow \infty}\left|\left\langle T_{j}, \varphi\right\rangle\right|=|\langle T, \varphi\rangle| \leq \gamma_{\alpha}(E) .
$$

To show (28), set $k_{\alpha}(x)=1 /|x|^{n-\alpha}$ and $f_{j}^{i}=x_{i} /|x|^{1+\alpha} * T_{j}, 1 \leq i \leq n$. We will treat first the case $n$ odd and of the form $n=2 k+1$. By (12),

$$
\begin{aligned}
\left\langle T_{j}, \varphi\right\rangle & =c \sum_{i=1}^{n}\left\langle f_{j}^{i}, \Delta^{k} \partial_{i} \varphi * k_{\alpha}\right\rangle \\
& =c \sum_{i=1}^{n} \int f_{j}^{i}(x)\left(\Delta^{k} \partial_{i} \varphi * k_{\alpha}\right)(x) d x .
\end{aligned}
$$

We mention here that if $n=2 k$, then one argues in the same way, but one has to use another reproduction formula analogous to (12) for this case (see [Pr1, Lemma $3.1])$.

Passing to a subsequence, we can assume that for each $1 \leq i \leq n, f_{j}^{i} \longrightarrow f^{i}$ in the weak $*$ topology of $L^{\infty}\left(\mathbb{R}^{n}\right)$. Then, using the definition of the weak $*$ $L^{\infty}$-convergence and the fact that $\Delta^{k} \partial_{i} \varphi * k_{\alpha}$ is an $L^{1}$-function, we get

$$
\begin{aligned}
\lim _{j \rightarrow \infty}\left\langle T_{j}, \varphi\right\rangle & =c \lim _{j \rightarrow \infty} \sum_{i=1}^{n} \int f_{j}^{i}(x)\left(\Delta^{k} \partial_{i} \varphi * k_{\alpha}\right)(x) d x \\
& =c \sum_{i=1}^{n} \int f^{i}(x)\left(\Delta^{k} \partial_{i} \varphi * k_{\alpha}\right)(x) d x .
\end{aligned}
$$

Define the distribution $T$ by

$$
\langle T, \varphi\rangle=c \sum_{i=1}^{n} \int f^{i}(x)\left(\Delta^{k} \partial_{i} \varphi * k_{\alpha}\right)(x) d x .
$$


Now we want to show that for $1 \leq i \leq n, f^{i}=x_{i} /|x|^{1+\alpha} * T$. For that we regularize $f_{j}^{i}$ and $T_{j}$. Take $\chi \in \mathcal{C}_{0}^{\infty}(B(0,1))$ with $\int \chi(x) d x=1$ and set $\chi_{\varepsilon}(x)=$ $\varepsilon^{-n} \chi(x / \varepsilon)$. Then we have, as $j \rightarrow \infty$,

$$
\left(\chi_{\varepsilon} * \frac{x_{i}}{|x|^{1+\alpha}} * T_{j}\right)(x)=\left(\chi_{\varepsilon} * f_{j}^{i}\right)(x) \longrightarrow\left(\chi_{\varepsilon} * f^{i}\right)(x), \quad x \in \mathbb{R}^{n},
$$

because $f_{j}^{i}$ converges to $f^{i}$ weak $*$ in $L^{\infty}\left(\mathbb{R}^{n}\right)$. On the other hand, since $\chi_{\varepsilon} * \frac{x_{i}}{|x|^{1+\alpha}} \in$ $\mathcal{C}^{\infty}\left(\mathbb{R}^{n}\right)$ and $T_{j}$ tends to $T$ in the weak topology of distributions, with controlled supports, we have

$$
\left(\chi_{\varepsilon} * \frac{x_{i}}{|x|^{1+\alpha}} * T_{j}\right)(x) \longrightarrow\left(\chi_{\varepsilon} * \frac{x_{i}}{|x|^{1+\alpha}} * T\right)(x), \quad x \in \mathbb{R}^{n} .
$$

Hence

$$
\chi_{\varepsilon} * \frac{x_{i}}{|x|^{1+\alpha}} * T=\chi_{\varepsilon} * f^{i}, \quad \varepsilon>0,
$$

and so, letting $\varepsilon \rightarrow 0, \frac{x_{i}}{|x|^{1+\alpha}} * T=f^{i}$.

\section{Sketch of the proof of Theorem 1}

This section will be devoted to the proof of inequality (41), namely

$$
\gamma_{\alpha}(E) \leq C \gamma_{\alpha,+}(E)
$$

We will adapt the line of reasoning in [T1] and [T2], where Tolsa proves the semiadditivity of analytic capacity and continuous analytic capacity respectively. We will also use the modifications introduced in [Vo], where the semiadditivity of Lipschitz harmonic capacity is proven (see also [RT]).

In fact, when one analyzes the proofs of [T1], [T2] and $[\mathrm{VO}$ ] one realizes that they depend on two technical facts, the exterior regularity property of $\gamma_{\alpha}$ (see Lemma 7) and an $L^{\infty}$-localization result, which is Lemma 5 in our setting. We must mention that the positivity properties of the symmetrization method for the Cauchy kernel discovered in [Me and [MeV] are an important ingredient for the proofs of [T1] and [T2. In Vo one has to circumvent this lack of positivity and modify Tolsa's idea.

We will explain now how each of the above-mentioned main ingredients takes part in the proof of (4): As we proved in Lemma 7, the capacities $\gamma_{\alpha}, 0<\alpha<n$, enjoy the exterior regularity property. This is also true for the capacities $\gamma_{\alpha,+}$, $0<\alpha<n$, just by the weak $\star$ compactness of the set of positive measures having total variation not exceeding 1 . We therefore can approximate a general compact set $E$ by sets which are finite unions of cubes of the same side length in such a way that the capacities $\gamma_{\alpha}$ and $\gamma_{\alpha,+}$ of the approximating sets are as close as we wish to those of $E$. Thus we can assume, without loss of generality, that $E$ is a finite union of cubes of the same size. This will allow us to implement an induction argument on the size of certain ( $n$-dimensional) rectangles. The first step involves rectangles of diameter comparable to the side length of the cubes whose union is $E$.

The starting point of the general inductive step in the proof of Tolsa's Theorem in [T1] (and [T2]) and in [Vo for the Lipschitz harmonic capacity case, consists in the construction of a positive Radon measure $\mu$ supported on a compact set $F$ which approximates $E$ in an appropriate sense. The construction of $F$ and $\mu$ gives readily that

$$
\gamma_{\alpha}(E) \leq C \mu(F)
$$


and

$$
\gamma_{\alpha,+}(F) \leq C \gamma_{\alpha,+}(E)
$$

which tells us that $F$ is not too small but also not too big. However, one cannot expect, in the context of [T1] and [T2, the Cauchy singular integral to be bounded on $L^{2}(\mu)$. In our case one cannot expect the $\alpha$-Riesz operator $R(\mu)$ to be bounded on $L^{2}(\mu)$. One has to carefully look for a compact subset $G$ of $F$ such that:

- $\mu(F) \leq C \mu(G)$.

- The restriction $\mu_{G}$ of $\mu$ to $G$ has $\alpha$-growth.

- The operator $R\left(\mu_{G}\right)$ is bounded on $L^{2}\left(\mu_{G}\right)$ with dimensional constants.

Moreover, recall from (7) that one has

$$
C^{-1} \gamma_{\alpha, o p}(E) \leq \gamma_{\alpha,+}(E) \leq C \gamma_{\alpha, o p}(E) \text {. }
$$

This completes the proof because then

$$
\begin{aligned}
\gamma_{\alpha}(E) & \leq C \mu(F) \leq C \mu(G) \leq C \gamma_{\alpha, o p}(G) \leq C \gamma_{\alpha, o p}(F) \\
& \leq C \gamma_{\alpha,+}(F) \leq C \gamma_{\alpha,+}(E) \leq C \gamma_{\alpha, o p}(E) .
\end{aligned}
$$

In [T1, T2 and $[\mathrm{Vo}$ the set $F$ is defined as the union of a special family of cubes $\left\{Q_{i}\right\}_{i=1}^{N}$ that cover the set $E$ and approximate $E$ at an appropriate intermediate scale. One then sets

$$
F=\bigcup_{i=1}^{N} Q_{i} .
$$

The construction of the set $F$ is different in the analytic capacity case and in the Lipschitz harmonic capacity case. In Tolsa's proof this construction is performed by using the positivity properties of the symmetrization of the Cauchy kernel discovered in $[\mathrm{Me}$ and $\mathrm{MeV}$. In our setting, the symmetrization of the Riesz kernels $x /|x|^{1+\alpha}$ only gives a positive quantity for $0<\alpha \leq 1$ (see [Pr1, Lemma 4.2]); therefore we have to circumvent the use of this positivity property and therefore modify Tolsa's idea. For this modification we will follow chapter 5 of $[\mathrm{Vo}$, where this was done for the Lipschitz harmonic capacity case, namely for $\alpha=n-1$. In fact the arguments in chapter 5 of $\mathrm{VO}$ are written for more general Calderón-Zygmund kernels of homogeneity $-\alpha$ and so they also work in our setting. Therefore, the construction of the approximating set $F$ with properties (29) and (30) can be done just as in [Vo, ch. 5].

By the definition of the capacity $\gamma_{\alpha}$ it follows that there exists a real distribution $T_{0}$ supported on the compact set $E$ such that:

(1) $\left|\left\langle T_{0}, 1\right\rangle\right| \geq \frac{\gamma_{\alpha}(E)}{2}$.

(2) $T_{0}$ has $\alpha$-growth and $G_{\alpha}\left(T_{0}\right) \leq 1$.

(3) $\left\|\frac{x_{j}}{|x|^{1+\alpha}} * T_{0}\right\|_{\infty} \leq 1, \quad 1 \leq j \leq n$.

The construction of $\mu$ is performed simultaneously with that of a real measure $\nu$, which should be viewed as a model for $T_{0}$ adapted to the family of cubes $\left\{Q_{i}\right\}_{i=1}^{N}$. For each cube $Q_{i}$ choose a ball $B_{i}$ concentric with $Q_{i}$ with radius $r_{i}$ comparable to $\gamma_{\alpha}\left(E \cap 2 Q_{i}\right)$ and set

$$
\mu=\sum_{i=1}^{N} \frac{r_{i}^{\alpha}}{\mathcal{L}^{n}\left(B_{i}\right)} \mathcal{L}_{\mid B_{i}}^{n}
$$


Consider now functions $\varphi_{i} \in \mathcal{C}_{0}^{\infty}\left(2 Q_{i}\right), 0 \leq \varphi_{i} \leq 1,\left\|\partial^{s} \varphi_{i}\right\|_{\infty} \leq C l\left(Q_{i}\right)^{-|s|}$, and $\sum_{i=1}^{N} \varphi_{i}=1$ on $\bigcup_{i} Q_{i}$. The measure $\nu$ is defined as

$$
\nu=\sum_{i=1}^{N} \frac{\left\langle T_{0}, \varphi_{i}\right\rangle}{\mathcal{L}^{n}\left(B_{i}\right)} \mathcal{L}_{\mid B_{i}}^{n}
$$

with $\mathcal{L}^{n}$ being the $n$-dimensional Lebesgue measure.

Notice that $\operatorname{supp}(\nu) \subset \operatorname{supp}(\mu) \subset F$. Moreover we have $d \nu=b d \mu$, with $b=\frac{\left\langle T_{0}, \varphi_{i}\right\rangle}{r_{i}^{\alpha}}$ on $B_{i}$. At this point, we need to show that our function $b$ is bounded to apply later a suitable $T(b)$ theorem. To estimate $\|b\|_{\infty}$ we need the localization Lemma 5. proved in section 3.2, which gives us

$$
\left\|\frac{x_{j}}{|x|^{1+\alpha}} * \varphi_{i} T_{0}\right\|_{\infty} \leq C, \quad 1 \leq j \leq n .
$$

We therefore obtain, by the definition of $\gamma_{\alpha}$,

$$
\left|\left\langle T_{0}, \varphi_{i}\right\rangle\right| \leq C \gamma_{\alpha}\left(2 Q_{i} \cap E\right), \quad \text { for } 1 \leq i \leq N .
$$

Hence $\|b\|_{\infty} \leq C$. It is now easy to see why $\gamma_{\alpha}(E) \leq C \mu(F)$ :

$$
\gamma_{\alpha}(E) \leq 2\left|\left\langle T_{0}, 1\right\rangle\right| \leq 2 \sum_{i=1}^{N}\left|\left\langle T_{0}, \varphi_{i}\right\rangle\right| \leq C \sum_{i=1}^{N} \gamma_{\alpha}\left(E \cap 2 Q_{i}\right)=C \mu(F) .
$$

Now everything is ready to apply a suitable variant of the $T(b)$ theorem (see NTVO). There is still one more difficulty: in applying the Nazarov, Treil and Volberg $T(b)$-type theorem, one needs to find a substitute for what they call the suppressed operators. It was already explained in Pr1 that there are at least two versions of such operators for the Riesz kernels that work appropriately (see [Pr1, (2.7) and (2.13)]).

\section{ACKNOWLEDGEMENTS}

The author was partially supported by grants 2009-SGR-420 (Generalitat de Catalunya) and MTM2007-60062 (Spanish Ministry of Science).

\section{REFERENCES}

[AH] D. R. Adams and L. I. Hedberg, Function Spaces and Potential Theory, Grundl. Math. Wiss. 314, Springer-Verlag, Berlin, 1996. MR1411441 (97j:46024)

[ENVo] V. Eidermann, F. Nazarov and A. Volberg, Vector-valued Riesz potentials: Cartan type estimates and related capacities, Proc. Amer. Math. Soc. (2010), 1-32. MR 2734959 (2011j:42020)

[EVo] V. Eidermann and A. Volberg, $L^{2}$-norm and estimates from below for Riesz transforms on Cantor sets, Preprint 2010.

[G] J. Garnett, Analytic Capacity and Measure, Lecture Notes in Math. 297, SpringerVerlag, Berlin, 1972. MR0454006 (56:12257)

[MPrV1] J. Mateu, L. Prat and J. Verdera, The capacity associated to signed Riesz kernels, and Wolff potentials, J. reine angew. Math. 578 (2005), 201-223. MR2113895 (2005m:31012)

[MPrV2] J. Mateu, L. Prat and J. Verdera, The capacity associated with scalar Riesz kernels, and analytic capacity, to appear in Indiana Univ. Math. J.

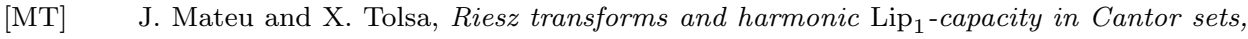
Proc. London Math. Soc. (3) 89 (2004), no. 3, 676-696. MR2107011 (2005g:42038)

[MaP] P. Mattila and P. V. Paramonov, On geometric properties of harmonic Lip - $_{1}$-apacity, Pacific J. Math. 171 (1995), no 2, 469-491. MR1372240 (97b:31005) 
[MzS] V. G. Maz'ya and T. O. Shaposhnikova, Theory of multipliers in spaces of differentiable functions, Monographs and Studies in Mathematics 23. Pitman (Advanced Publishing Program), Boston, MA, 1985. MR785568 (87j:46074)

[Me] M. S. Melnikov, Analytic capacity: discrete approach and curvature of measure, Sbornik: Mathematics 186 (1995), no. 6, 827-846. MR1349014(96f:30020)

$[\mathrm{MeV}] \quad$ M. S. Melnikov and J. Verdera, A geometric proof of the $L^{2}$ boundedness of the Cauchy integral on Lipschitz graphs, Inter. Math. Res. Not. 7 (1995), 325-331. MR.1350687 (96f:45011)

[NTVo] F. Nazarov, S. Treil and A. Volberg, The T(b)-theorem on non-homogeneous spaces that proves a conjecture of Vitushkin, CRM preprint, December 2002.

[P] P. V. Paramonov, On harmonic approximation in the $\mathcal{C}^{1}$-norm, Math. USSR Sbor. 71 (1992), no. 1. MR1085885 (92j:31006)

[Pr1] L. Prat, Potential theory of signed Riesz kernels: capacity and Hausdorff measure, Intern. Math. Res. Not. 19 (2004), 937-981. MR2037051(2004k:31012)

[Pr2] L. Prat, Null sets for the capacity associated to Riesz kernels, Illinois Journal of Math. 48 (2004), no. 3, 953-963. MR2114262 (2005h:42040)

[RT] A. Ruiz de Villa and X. Tolsa, Characterization and semiadditivity of the $C^{1}$ harmonic capacity, Trans. Amer. Math. Soc. 362 (2010) 3641-3675. MR2601603 (2011g:31008)

[St] E. M. Stein, Singular integrals and differentiability properties of functions, Princeton University Press, Princeton, 1970. MR0290095 (44:7280)

[T1] X. Tolsa, Painlevé's problem and the semiadditivity of analytic capacity, Acta Math. 190 (2003), no. 1, 105-149. MR1982794 (2005c:30020)

[T2] X. Tolsa, The semiadditivity of continuous analytic capacity and the inner boundary conjecture, Amer. J. Math. 126 (2004), 523-567. MR2058383 (2005f:30052)

[T3] X. Tolsa, Calderón-Zygmund capacities and Wolff potentials on Cantor sets, Journal of Geometric Analysis 21 (2011), 195-223. MR2755682

[V] J. Verdera, $\mathcal{C}^{m}$ approximation by solutions of elliptic equations, and Calderón-Zygmund operators, Duke Math. J. 55 (1987), 157-187. MR883668 (88e:35011)

[Vo] A. Volberg, Calderón-Zygmund capacities and operators on nonhomogeneous spaces, CBMS Reg. Conf. Ser. Math. 100. Amer. Math. Soc., Providence, RI, 2003. MR2019058 (2005c:42015)

Departament de Matemàtiques, Universitat Autònoma de Barcelona, 08193 Bellaterra (BArcelona), Catalunya

E-mail address: laurapb@mat.uab.cat 